\title{
Costotransversectomy in thoracic spinal tuberculosis
}

\author{
AH Botha MMed(Ortho), FCS(Ortho) \\ Spinal Surgery Fellow, Groote Schuur Hospital \\ JH Davis MMed(Ortho), FCS(Ortho) \\ Senior Consultant Tygerberg Academic Hospital; Head of Spinal Surgery Services
}

\author{
Corresponding author: \\ Dr AH Botha \\ 5 Woodlands \\ Somerset West \\ 7130 \\ Email: attiebotha@gmail.com \\ Tel: 021-8545852
}

\begin{abstract}
Background: The escalating global pandemic of tuberculosis infections results in 8 million new cases diagnosed each year. The thoracic and thoracolumbar spine is the most prevalent area involved in musculoskeletal tuberculosis. Deformity with associated neurological compromise, requiring extended in-patient treatment and rehabilitation, is common.

Multidrug-resistant tuberculosis is prevalent and tissue samples are needed to obtain bacterial culture and sensitivity. Decompression of the spinal canal, directly or indirectly, should accelerate neurological recovery.

Methods: A retrospective study was performed at Tygerberg Hospital to evaluate the efficacy of costotransversectomy in spinal thoracic tuberculosis with regard to neurological recovery and deformity. Neurological status was compared at 6 months post-operatively with the pre-surgical status. The end deformity was compared with Rajasakeran's equation.

Results: Thirty patients met the inclusion criteria, with an average age of 37 years. Fifteen patients were HIV positive, and ten of them on highly active antiretroviral therapy (HAART). The average CD4 count was 235.

The mean neurological status of the group was classified as Frankel C, but this improved to Frankel D at 6 months post-surgery. Initial sagittal deformity was $18.7^{\circ}$, which increased to $26^{\circ}$ one year post-operatively. This was not significantly different from the $25.6^{\circ}$ kyphosis predicted by the Rajasekaran formula.

A $67 \%$ positive culture yield for TB was obtained which compared favourably to percutaneous transpedicular needle biopsies performed at the same institute which had a yield of $56 \%$.

Conclusions: Costotransversectomy is a simple procedure resulting in indirect decompression of the spinal cord, improving the microbiological diagnosis of spinal tuberculosis, and possibly leading to earlier neurological recovery, without the risk of creating further instability and greater deformity.
\end{abstract}

Key words: tuberculosis, thoracic spine, costotransversectomy, deformity spine surgery

http:/ / dx.doi.org/10.17159/2309-8309/2016/v15n1a10

\section{Introduction}

The escalating global pandemic of tuberculosis infections results in 8 million new cases diagnosed each year. ${ }^{1-4}$ Two billion people (or a third of the of the world's population) have been or are currently infected with tuberculosis.

The prevalence of HIV results in a high incidence of musculoskeletal tuberculosis, of which $60 \%$ affects the skeleton, and in 50\% the spine ${ }^{1,46}$ with the thoracic and thoracolumbar areas most commonly affected (Figure 1). This often results in deformity with associated neurological compromise, requiring extended in-patient treatment and rehabilitation.
Tissue diagnosis and antimicrobial sensitivity is crucial given the increased prevalence of extreme drug-resistant (XDR) and multidrug-resistant (MDR) tuberculosis ${ }^{1}$ reported in up to $12 \%$ of cases in South Africa. ${ }^{2,7}$

Percutaneous needle biopsy does not decompress the spinal canal, and neurologic recovery is dependent on medical treatment alone. Costotransversectomy is a relatively simple procedure that yields a good tissue sample while indirectly decompressing the spinal canal simultaneously, theoretically leading to earlier neurological recovery.

A potential concern however, is the structural integrity of the spinal column, and the possible adverse effect on spinal stability of the removal of the transverse process, underlying 
rib-head and costovertebral joint resulting in a greater end deformity.

We present a series of 30 patients who underwent costotransversectomy for thoracic spinal tuberculosis with a predicted final kyphosis less than $70^{\circ}$.

\section{Materials and methods}

A retrospective case note and radiological record review was performed of all patients treated during the period from 1 January 2010 to 30 June 2012 at Tygerberg Hospital, presenting with suspected thoracic or thoracolumbar spinal tuberculosis, and in whom the final predicted deformity was less than $70^{\circ}$ kyphosis. All patients with records included in our cohort underwent an indirect decompression and biopsy through costotransversectomy.

The records of the patients included were reviewed for demographic data, HIV status, neurological status and initial spinal deformity.

At the 6 months post-operative mark, the neurological status of the patient was re-assessed and compared to the neurologic status pre-operatively.

One year post-operatively, the deformity was assessed and compared to the final deformity predicted by Rajasekaran's equation as calculated from the presenting standing plainfilm radiographs. ${ }^{8}$

Thirty patients were included in the study, 16 male and 14 female. The average age was 37 years (6-67 years \pm 15$)$. Fifteen patients were HIV positive with an average CD4 count of 235 (10-500 \pm 157$)$.

Ten of these patients were on highly active anti-retroviral therapy (HAART). Average ESR was $70(12-125 \pm 33)$ presurgery, and improved on average to 28 at one year postoperatively $(10-53 \pm 12)$. Polymerase chain reaction (PCR) testing was not performed on all of these patients.

\section{Results}

The average pre-operative Frankel grade was C, with 17 patients having a Frankel grade A, B or C, equating to a nonambulatory patient requiring prolonged in-patient treatment and extensive rehabilitation. Thirteen patients were independently mobile.

At 6 months post-operatively the average Frankel grade improved to a grade D, with only six patients still rated as Frankel Grade A, B or C. The remaining 24 patients were independently mobile with Frankel Grades of D or E, and no longer required in-patient treatment. This improvement was statistically significant $(\mathrm{p}=0.01)$ (Table I). The neurological recovery of the HIV-positive and MDR-TB group was not statistically different from the HIV-negative group.

Tissue cultures were positive in 20 patients after 22.9 days (67\% yield) (range 13-47 \pm 9.5). Three patients $(10 \%)$ cultured multidrug-resistant tuberculosis. The demonstrated yield proved superior to percutaneous needle biopsies performed at the same institution for the same time period, with a positive culture yield of $56 \%$ reported. $^{2}$

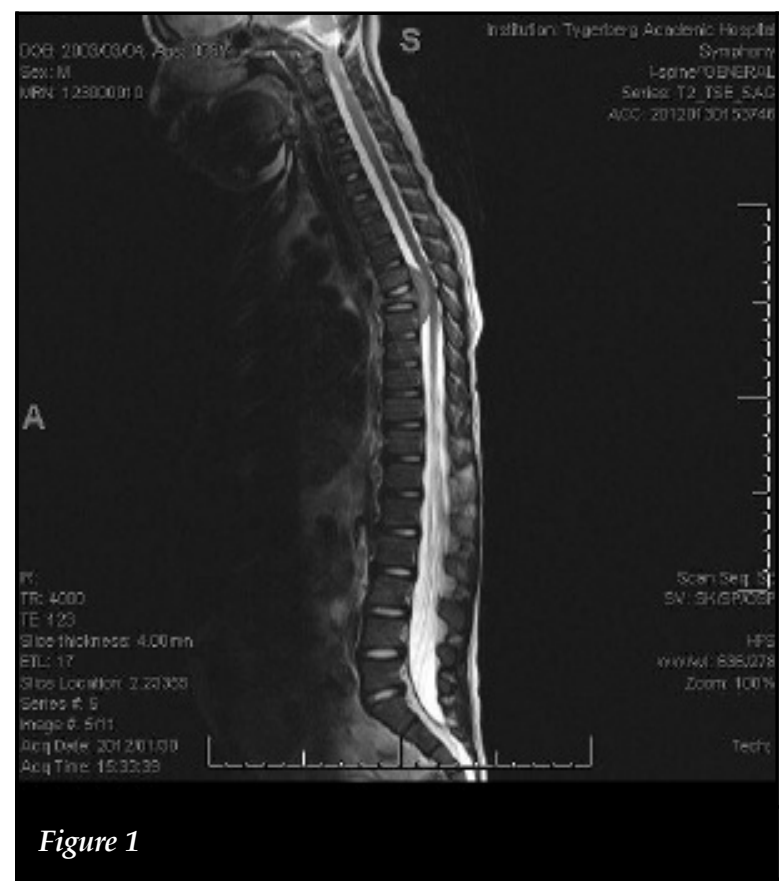

Initial radiographical analysis revealed an average kyphotic deformity of $18.7^{\circ}$ (range $4-37^{\circ} \pm 9$ ). Rajasekaran's equation of $Y=5.5^{\circ}+30.5 \mathrm{x}$, where $\mathrm{Y}$ is the final predicted deformity and $x$ the amount of vertebral body involved in tenths, was used to predict the final deformity. ${ }^{8}$ The average amount of vertebral body involved was 0.65 , giving a predicted end deformity of $25.6^{\circ} \pm 11.5$ in the sagittal plane. The sagittal deformity one year post-operatively was $26^{\circ} \pm 10.2^{\circ}$ (Table II). This was not significantly different from the final predicted kyphosis.

The initial coronal deformity was on average $3.7^{\circ}$ (range $\left.0-19^{\circ} \pm 4.9\right)$ and at one year follow-up the average was $4.2^{\circ} \pm 5.2^{\circ}$.

No complications were encountered in this series.

Table I: Frankel Grade of neurological involvement

\begin{tabular}{|c|c|c|}
\hline Frankel Grade & Pre-operative & Post-operative \\
\hline A & 4 & 0 \\
\hline B & 2 & 3 \\
\hline C & 11 & 3 \\
\hline D & 7 & 8 \\
\hline $\mathrm{E}$ & 6 & 16 \\
\hline
\end{tabular}

Table II: Deformity pre-operatively and one year postoperatively

\begin{tabular}{|l|c|c|}
\hline \multicolumn{1}{|c}{ Pre-operative } & Post-operative \\
\hline Coronal & $3.7^{\circ}$ & $4.2^{\circ}$ \\
\hline Sagittal & $18.7^{\circ}$ & $26^{\circ}$ \\
\hline $\begin{array}{l}\text { Predicted sagittal } \\
\text { deformity }\end{array}$ & & $25.6^{\circ}$ \\
\hline
\end{tabular}




\section{Discussion}

In 2010 there were globally 8.8 million new cases of TB diagnosis, in part due to the prevalence of $\mathrm{HIV}^{9,10}$ Cases with multi- and extreme drug-resistant tuberculosis make bacterial culture and antimicrobial sensitivity mandatory before initiating treatment.

Neurological deficit can be as high as $41 \%$, especially when it occurs in the thoracic or thoracolumbar area. ${ }^{14,69,911}$ Many of these patients are bed-bound due to paraplegia, requiring prolonged in-hospital stay and extended rehabilitation at an appropriate facility. This places an additional burden on an already strained health care system.

Much has been written about the treatment and surgical management of spinal tuberculosis. ${ }^{6,10,12-18}$ Hodgkin and Stock advocated aggressive surgical debridement of all tuberculous lesions in the spine. ${ }^{17}$ They advocated that anti-tuberculous chemotherapy did not penetrate the osseous anatomy adequately, and only surgical debridement removed the necrotic tissue. Tuli advocated the 'middle-path' regimen, where medical treatment is the cornerstone of treatment and surgery is only advocated for selected cases. ${ }^{18}$

The Medical Research Council's (MRC) Working Party on spinal tuberculosis showed that medical management of spinal tuberculosis should be the cornerstone of treatment. ${ }^{19-22}$ The MRC's 15-year follow-up report states that $87 \%$ of the study patients attained a favourable status (defined as: no evidence of CNS involvement, no sinus or clinically evident abscess, radiological quiescence of the disease and no restriction of physical activity), regardless of surgical treatment modality. ${ }^{20}$

It is evident from the MRC's research that radical debridement and reconstruction should be reserved for select cases due to the resource-intensive nature of these procedures. Reconstruction should be reserved for cases with a predicted end deformity greater than $70^{\circ}$, continued neurologic deterioration or instability.

Although neurological recovery happens with chemotherapy alone, there is very little literature available regarding the time it takes a patient to achieve mobility to such a degree that they can be managed as an outpatient.

Tissue diagnosis and testing for resistance is mandatory given the incidence of MDR and XDR TB. ${ }^{7}$ Currently percutaneous transpedicular needle is still the most commonly used modality to obtain tissue for microscopy, culture and sensitivity (MCS). No decompression of the spinal cord is performed either directly or indirectly, and neurological recovery is dependent on chemotherapy alone. Our study group had a $67 \%$ yield for positive culture, with reported yields from needle biopsies ranging from $42-76 \% .7,73,24$

The current policy at Tygerberg Hospital's spinal unit is to perform a costotransversectomy for patients with suspected thoracic spinal tuberculosis with a predicted end deformity of less than $70^{\circ}$. It is a relatively simple procedure that yields a good amount of tissue under direct vision, as well as a resultant indirect decompression of the spinal cord.
Patients are positioned prone on a Relton-Hall frame in the same position as for a percutaneous transpedicular needle biopsy. The correct level is identified with fluoroscopy, and a midline incision is made. The costovertebral junction is then identified, the transverse process and the underlying rib head is resected towards the tubercle of the rib. Following removal of the rib head, the periosteum is then elevated from the vertebral body while staying extra-pleural until pus or necrotic material is encountered.

An adequate specimen can then be sampled under direct vision, and the spinal cord is decompressed indirectly by means of decompressing the abscess cavity and allowing pus to drain from the spinal canal.

We were able to demonstrate that $80 \%$ (24 patients) of our patients were ambulatory 6 months post-operatively versus $43 \%$ (13 patients) pre-operatively. This finding was statistically significant, $\mathrm{p}=0.01$. The ambulatory patients did not require any in-patient treatment at a rehabilitation facility.

Our $67 \%$ positive culture on specimens compared favourably to national and international standards as mentioned earlier. ${ }^{2}$ Costotransversectomy compared favourably to the $56 \%$ yield of needle biopsies performed at the same institution for suspected thoracolumbar and lumbar spinal tuberculosis. ${ }^{2}$ This was attributed to the processing of the samples at the laboratory where all specimens were decontaminated before culture and microscopy.

Removal of the rib head has been postulated to decrease the stability of the spinal column which would in turn increase the deformity. Our study shows that when compared to the final predicted sagittal deformity as per Rajasekaran's equation, there was no statistically significant increase in the sagittal deformity. ${ }^{8}$

In summary, costotransversectomy provides indirect decompression of the thoracic spinal canal possibly leading to earlier neurological recovery and a decreased need for inpatient treatment. The yield with regard to microbiological diagnosis obtained is acceptable when compared to international literature. Using Rajasekaran's equation we also showed that removing the rib head does not worsen, or improve the deformity.

Costotransversectomy for thoracic spinal tuberculosis is an effective treatment option in thoracic spinal tuberculosis, both in establishing diagnosis and appropriate treatment sensitivities, as well as the additional benefit of potentially earlier neurological recovery.

\section{Conclusion}

Indirect decompression via costotransversectomy provides a simple option for cord decompression and simultaneous confirmation of diagnosis and sensitivity to anti-microbials, in the sub-group of patients with an acceptable predicted end deformity. A statistically significant improvement could be demonstrated at six months $(p=0.01)$ with regard to neurological deficit. 
No detrimental effect could be shown in progression of spinal deformity.

\section{Conflict of interest statement}

The article is the original work of the authors and no funds were received in respect of the contents.

\section{References}

1. Jain AK, Kumar J. Tuberculosis of spine: neurological deficit. European Spine Journal: official publication of the European Spine Society, the European Spinal Deformity Society, and the European Section of the Cervical Spine Research Society 2013;22 Suppl 4:624-33.

2. Watt JP, Davis JH. Percutaneous core needle biopsies: The yield in spinal tuberculosis. SAMJ: South African Medical Journal 2014;104:29-32.

3. Millet J-P, Moreno A, Fina L, et al. Factors that influence current tuberculosis epidemiology. European Spine Journal 2013;22:539-48.

4. Jain A. Tuberculosis of the spine a fresh look at an old disease. Journal of Bone E Joint Surgery (Brit) 2010;92:905-13.

5. Tuli SM. Historical aspects of Pott's disease (spinal tuberculosis) management. European Spine Journal 2013;22 Suppl 4:529-38.

6. Tuli SM. Tuberculosis of the spine: a historical review. Clin Orthop Relat Res 2007;460:29-38.

7. Dunn R. The medical management of spinal tuberculosis. SA Orthopaedic Journal 2010;9:37-41.

8. Rajasekaran S, Shanmugasundaram TK. Prediction of the angle of gibbus deformity in tuberculosis of the spine. Journal of Bone and Joint Surgery (Am) 1987;69:503-509.

9. Jain AK, Dhammi IK. Tuberculosis of the spine: a review. Clin Orthop Relat Res 2007;460:39-49.

10. Turgut M. Spinal tuberculosis (Pott's disease): its clinical presentation, surgical management, and outcome. A survey study on 694 patients. Neurosurg Rev 2001;24:8-13.

11. Ferrer MF, Torres LG, Ramírez OA, Zarzuelo MR, del Prado González N. Tuberculosis of the spine. A systematic review of case series. International Orthopaedics 2012;36:221-31.

12. Mak KC, Cheung KM. Surgical treatment of acute TB spondylitis: indications and outcomes. European Spine Journal 2013;22 Suppl 4:603-11.

13. Jain AK, Jain S. Instrumented stabilization in spinal tuberculosis. Int Orthop 2012;36:285-92.

14. Ozdemir HM, Us AK, Ogun T. The role of anterior spinal instrumentation and allograft fibula for the treatment of pott disease. Spine (Phila Pa 1976) 2003;28:474-79.
15. Faraj AA. Anterior instrumentation for the treatment of spinal tuberculosis. Journal of Bone and Joint Surgery (Am) 2001;83-A:463-64.

16. Upadhyay SS, Saji MJ, Yau AC. Duration of antituberculosis chemotherapy in conjunction with radical surgery in the management of spinal tuberculosis. Spine (Phila Pa 1976) 1996;21:1898-903.

17. Tenth report of the Medical Research Council Working Party on Tuberculosis of the Spine. A controlled trial of six-month and nine-month regimens of chemotherapy in patients undergoing radical surgery for tuberculosis of the spine in Hong Kong. Tubercle 1986;67:243-59.

18. Tuli S. Results of treatment of spinal tuberculosis by 'middle-path' regime. Journal of Bone \& Joint Surgery (Brit) 1975;57:13-23.

19. Fox W, Ellard GA, Mitchison DA. Studies on the treatment of tuberculosis undertaken by the British Medical Research Council tuberculosis units, 1946-1986, with relevant subsequent publications. International Journal of Tuberculosis and Lung Disease 1999;3:S231-79.

20. Darbyshire J. A 15-year assessment of controlled trials of the management of tuberculosis of the spine in Korea and Hong Kong - Thirteenth Report of the Medical Research Council Working Party on Tuberculosis of the Spine. Journal of Bone and Joint Surgery (Brit) 1998;80:456-62.

21. Smith M. Five-year assessment of controlled trials of inpatient and outpatient treatment and of plaster of Paris jacket for tuberculosis of the spine in children on standard chemotherapy: Fifth Report of the Medical Research Council Working Party on Tuberculosis of the Spine. J Bone Joint Surg (Br) 1976 Nov;58:339-411.

22. Working Party on Tuberculosis of the Spine. A five-year assessment of controlled trials of in-patient and out-patient treatment and of plaster-of-Paris jackets for tuberculosis of the spine in children on standard chemotherapy. J Bone Joint Surg (Br) 1976:399-41.

23. Colmenero JD, Ruiz-Mesa JD, Sanjuan-Jimenez R, Sobrino B, Morata P. Establishing the diagnosis of tuberculous vertebral osteomyelitis. European Spine Journal 2013;22 Suppl 4:579-86.

24. Merino P, Candel FJ, Gestoso I, Baos E, Picazo J. Microbiological diagnosis of spinal tuberculosis. Int Orthop 2012;36:233-38

This article is also available online on the SAOA website (www.saoa.org.za) and the SciELO website (www.scielo.org.za). Follow the directions on the Contents page of this journal to access it. 